\title{
Early transoesophageal echocardiography in cryptogenic and lacunar stroke and transient ischaemic attack
}

Bruno Censori, Francesca Colombo, Maria Grazia Valsecchi, Laura Clivati, Anna Zonca, Massimo Camerlingo, Luciano Casto, Mario Salvatore De Tommasi, Angelo Mamoli

\begin{abstract}
Objectives-To test the hypothesis that transoesophageal echocardiography (TOE) carried out within three days of a first stroke or transient ischaemic attack of cryptogenic or lacunar type may disclose more thrombi or spontaneous echo contrast (SEC) than previously reported. This finding may help early treatment decisions.
\end{abstract}

Methods-Patients aged between 40 and 80 years, admitted for transient ischaemic attack or ischaemic stroke during a 40 month period, were prospectively considered. TOE was carried out within 72 hours of symptom onset with a $5 \mathrm{MHz}$ biplanar transducer. Subjects with recurring events, very severe strokes, large artery obstructions, or obvious cardiac sources of embolism were excluded.

Results-Sixty five patients were studied, 43 with a cryptogenic stroke or transient ischaemic attack $(66.2 \%)$, and 22 with a lacunar stroke $(33.8 \%)$. The mean (SD) interval between symptom onset and TOE was 43.4 (17.2) hours for cryptogenic, and 48.5 (19.5) hours for lacunar patients. Atrial thrombi were found in one patient with a cryptogenic stroke $(2.32 \%$ of cryptogenic events; $95 \%$ confidence interval 0.06-12.29), whereas SEC was found in five patients $(7.7 \%$ overall), two with a lacunar and three with a cryptogenic stroke.

Conclusions-An early TOE does not seem to increase substantially the detection of atrial thrombi or SEC in patients with a first stroke or transient ischaemic attack of cryptogenic or lacunar nature. Therefore, this examination can be carried out when the patients' conditions are stable, and without overloading the cardiovascular laboratory daily schedule.

(F Neurol Neurosurg Psychiatry 1998;64:624-627)

Keywords: stroke; cerebral ischaemia, transient; echocardiography; transesophageal

Correspondence to: Dr Bruno Censori, Divisione Neurologia 2, Ospedali Riuniti, Largo Barozzi 1, 24100 Bergamo, Italy. Telephone 003935269 413; fax 003935269679 .

Received 29 July 1997 and in revised form 10 October 1997

Accepted 23 October 1997 in a very small percentage of these strokes. ${ }^{5-8}$ Much more commonly, TOE has provided images that only indirectly suggest a cardioembolic mechanism, such as a patent foramen ovale, ${ }^{5910}$ or atrial septal aneurysms, ${ }^{11}{ }^{12}$ or it has shown large aortic plaques, ${ }^{13}{ }^{14}$ that imply an artery to artery embolisation. Spontaneous echo contrast (SEC) is considered to be a strong marker of stasis and of thrombotic tendency in the left atrium or atrial appendage, ${ }^{15} 16$ but it is usually found in chronic atrial fibrillation or mitral stenosis, ${ }^{17}$ and is rare in strokes of undetermined origin. ${ }^{56}$ The relevance of other findings, such as Chiari's network ${ }^{18}$ or valve strands, ${ }^{19}{ }^{20}$ is still unclear.

In most studies on stroke patients the interval between symptom onset and TOE is either not specified or exceeds one week. ${ }^{5-7}{ }^{13}$ In some studies TOE was carried out within one week from the first symptoms, ${ }^{8}{ }^{21}$ but only one series reports a mean interval from embolisation shorter than three days. ${ }^{22}$ However, in this study several different emboligenic conditions were pooled together, including atrial fibrillation, and all patients were younger than 60 years.

Perhaps carrying out TOE very close to symptom onset would disclose more images that strongly suggest a cardiac embolism than reported, just as angiography carried out early after stroke onset shows more arterial obstructions than at later times. ${ }^{23}$ As days pass, a residual atrial or appendage clot may silently embolise to other organs, or may spontaneously lyse. Other conditions, such as SEC, might also disappear with time.

We report the results of a TOE investigation of first ever transient ischaemic attacks and strokes of undetermined origin or of lacunar type in patients older than 40 years, in which all TOEs were carried out within three days from symptom onset. Lacunar strokes were included because it has been hinted that at least some of them may be due to embolism from the heart. $^{524}$

\section{Patients and methods}

We have considered all ischaemic strokes or transient ischaemic attacks admitted to our stroke unit from May 1993 until December 1996. Our hospital is a primary care institution, and patients with cerebral ischaemia are admitted from the emergency room or directly from their homes, without any selection. Stroke and transient ischaemic attacks were defined according to standard criteria. ${ }^{25}$ Transient 
Table 1 General features

\begin{tabular}{|c|c|c|c|}
\hline & $\begin{array}{l}\text { Lacunar group } \\
(n=22)\end{array}$ & $\begin{array}{l}\text { Cryptogenic group } \\
(n=43)\end{array}$ & $\begin{array}{l}p \text { Value } \\
\left(\chi^{2}\right)\end{array}$ \\
\hline Age (mean (SD) y) & $66.5(9.5)$ & $63.4(10.9)$ & $\mathrm{NS}^{\star}$ \\
\hline Males (n (\%)) & $17 \quad(77.3)$ & $27 \quad(62.8)$ & NS \\
\hline Hypertension (n (\%)) & $14 \quad(63.6)$ & $17 \quad(39.5)$ & NS \\
\hline Diabetes (n (\%)) & $5 \quad(22.7)$ & $5 \quad(11.6)$ & NS \\
\hline Smoking (n (\%)) & $9 \quad(40.9)$ & $19(44.2)$ & NS \\
\hline Hypercholesterolaemia (n (\%)) & $3(13.6)$ & $(16.3)$ & NS \\
\hline $\operatorname{IHD}(\mathrm{n}(\%))$ & $(13.6)$ & $(9.3)$ & NS \\
\hline TIA (n (\%)) & 0 & (23.3) & 0.036 \\
\hline
\end{tabular}

${ }^{\star}$ Student's t test; IHD = ischaemic heart disease; TIA = transient ischaemic attack.

dizziness, isolated vertigo, diplopia, or loss of consciousness were not considered as transient ischaemic attacks.

Exclusion criteria were: (1) admission more than three days from onset of symptoms; (2) history of previous strokes or transient ischaemic attacks, in any territory; (3) age older than 80 years or younger than 40 ; (4) moderate to severe dementia before the stroke; (5) consciousness impairment or a very severe deficit, quantified by a Canadian neurological scale $(\mathrm{CNS})^{26}$ score $\leqslant 3$; (6) any contraindication to oral anticoagulation (severe uncontrolled hypertension, liver cirrhosis, malignant neoplasms, recent major neurosurgical interventions). All patients fulfilling admission criteria underwent routine blood tests, ECG, chest radiography, colour coded duplex study of neck vessels, and brain CT within 24 hours of admission. Brain CT was repeated five to 10 days after symptom onset, and the following features were noted: (1) presence and type (lacunar $v$ territorial) of one or more recent lesions; (2) presence and type of old, asymptomatic ischaemic lesions.

The aetiology of the ischaemic event was assigned to one of four categories: (1) large artery atherothrombosis, when a stenosis $\geqslant 50 \%$ or occlusion of the common or internal carotid artery ipsilateral to the stroke was present, (2) cardioembolism, when one of the high risk sources of cardioembolism of the TOAST classification was present, ${ }^{27}$ (3) lacune,$^{27}$ and (4) cryptogenic, when none of the first three categories applied.

Only patients with strokes or transient ischaemic attacks of the lacunar or undetermined type were considered. All patients underwent transthoracic (TTE) and TOE in one session. TTE was carried out with an Acuson 128XP/5 machine (Acuson, Mountain View, CA, USA), using a $2.5 \mathrm{MHz}$ or a 3.5 $\mathrm{MHz}$ transducer. Standard parasternal, apical, and subcostal views were obtained. In particular, TTE was used to assess the presence of mitral valve prolapse, ventricular thrombi, segmental wall motion abnormalities, and to determine the ejection fraction. TOE was carried out with the same machine as TTE, using a biplanar 5.0 MHz transducer. After fasting for four to six hours, the patient was premedicated with intravenous metoclopramide and oral diazepam one hour before TOE. The examination was performed with the patient in the left lateral decubitus; the introduction of the transducer was preceded by topical oropharyngeal anaesthesia with lidocaine spray. In all cases $10 \mathrm{ml}$ agitated normal saline were rapidly injected into an antecubital vein, at rest and during a Valsalva manoeuvre. The following features were noted on the TOE report: $(a)$ left atrial or left appendage thrombus; $(b)$ echo contrast in the left atrium or atrial appendage; SEC was defined as dynamic swirling echoes that persisted in different planes; $(c)$ patency of the foramen ovale; patent foramen ovale was diagnosed when there was evidence of right to left atrial communication at rest, or after contrast injection when $\geqslant$ three microbubbles passed from the right to the left atrium within three heart cycles of their appearance into the right atrium; (d) atrial septal aneurysm, defined as $>10 \mathrm{~mm}$. oscillation of the fossa ovalis membrane into the left or right atrium during the heart cycle; (e) protruding plaques $>4 \mathrm{~mm}$ thickness in the ascending aorta or aortic arch.

The interval between symptom onset and TOE was calculated in hours. For strokes that occurred during the night, the interval was calculated taking midnight as a conventional time of symptom onset.

Statistical analysis of the results was carried out using the $\chi^{2}$ test for categorical variables, and Student's $t$ test for continuous variables with a normal distribution.

\section{Results}

Sixty five patients underwent TOE according to the study protocol. They represented no more than $40 \%$ of all eligible patients admitted during the same period. The reasons why TOE was not carried out in all eligible patients were the unavailability of TOE or cardiologists, and referral to hospital too close to the expiration of the three day period. Three patients could not tolerate the introduction of the probe. The examination was well tolerated by all other patients, and no complications occurred.

The mean (SD) age of patients undergoing TOE was 64.5 (10.5) years; they were 44 men $(67.8 \%)$ and 21 women (32.3\%). Fifty five had had a stroke $(84.6 \%)$, and 10 had had a transient ischaemic attack $(15.4 \%)$. The ischaemic event was of undetermined origin in 43 patients $(66.2 \%)$, and of lacunar type in 22 $(33.8 \%)$. The vertebrobasilar or posterior cerebral artery territories were affected in 13 patients $(20.0 \%)$. Table 1 shows the features of the lacunar and cryptogenic groups. Stroke severity was generally mild to moderate, with a mean (SD) CNS score of 7.6 (1.5) points. No patients had recurrences of stroke or died during their stay in hospital. Five patients were being treated with intravenous heparin at the time of $\mathrm{TOE}(7.7 \%)$; three of them had activated partial thromboplastin time ratios $>1.5$.

The CT disclosed a recent lesion, consistent with symptoms, in 39 patients $(60.0 \%)$. Old lesions were present in 16 patients $(24.6 \%)$; they were of the lacunar type in 10 cases $(62.5 \%)$, and of territorial type in the other six (37.5\%).

Transthoracic echocardiography disclosed a small mitral calcification in one patient $(1.5 \%)$, mild mitral valve prolapse in one $(1.5 \%)$, small calcifications of the aortic valve in three 
Table 2 Transoesophageal echocardiography findings

\begin{tabular}{llcl}
\hline & $\begin{array}{l}\text { Lacunar group } \\
(n=22)\end{array}$ & $\begin{array}{l}\text { Cryptogenic group } \\
(n=43)\end{array}$ & $p$ Value $\left(\chi^{2}\right)$ \\
\hline Atrial/appendage thrombi & 0 & $1(2.3 \%)$ & NS \\
SEC & $2(9.1 \%)$ & $3(7.0 \%)$ & NS \\
PFO & $6(27.3 \%)$ & $17(39.5 \%)$ & NS \\
ASA & 0 & $2(4.6 \%)$ & NS \\
Aortic plaques & $1(4.5 \%)$ & $2(4.6 \%)$ & NS \\
\hline
\end{tabular}

$\mathrm{SEC}=$ spontaneous echo contrast $\mathrm{PFO}=$ patent foramen ovale; ASA = atrial septal aneurysm.

$(4.6 \%)$, and small mural akinesia in three $(4.6 \%)$. The ejection fraction was $>45 \%$ in all patients.

The mean (SD) interval between onset of syptoms and TOE was 45.2 (18.1) hours; it was 43.4 (17.2) hours for stroke-transient ischaemic attacks of undetermined origin, and 48.5 (19.5) hours for lacunar strokes (difference not significant). Echocardiography was carried out within 24 hours from symptom onset in eight patients (12.3\%), between 25 and 48 hours in 31 patients $(47.7 \%)$, and between 49 and 72 hours in 26 patients $(40.0 \%)$.

Table 2 shows all TOE findings. Overall, 30 patients had at least one abnormality $(46.2 \%)$, 22 in the cryptogenic $(51.2 \%)$, and eight in the lacunar group $(36.4 \%)$. A left atrial thrombus was detected in one patient, who had a stroke of undetermined origin $(2.32 \%$ of cryptogenic stroke-transient ischaemic attacks; $95 \%$ confidence interval 0.06-12.29). At CT this patient had an old territorial infarction of the right occipital lobe, of which he had never been aware, besides his recent lesion. Spontaneous echo contrast was seen in five cases $(7.7 \%)$, three with cryptogenic strokes $(7.0 \%)$, and two with lacunar strokes $(9.1 \%)$, and was always of a mild degree. The mean (SD) age of patients with SEC was higher than that of patients without SEC (70.2 (9.8) years $v 64.0$ (10.5) years), although not significantly, whereas the interval between symptoms and TOE was similar in SEC positive and SEC negative patients. A patent foramen ovale was present in 23 patients (35.4\% overall), six with a lacunar stroke $(27.3 \%)$, and 17 with a stroke or transient ischaemic attack of undetermined origin $(39.5 \%)$. An atrial septal aneurysm was seen in two patients, both with cryptogenic strokes ( $4.6 \%$ of cryptogenic ischaemias). Finally, aortic plaques protruding $>4 \mathrm{~mm}$ were seen in three patients $(4.6 \%)$, one with a lacunar stroke $(4.5 \%)$ and two with cryptogenic strokes $(4.6 \%)$.

Only the patient with the atrial thrombus was given oral anticoagulants at discharge.

\section{Discussion}

The hypothesis that a very early TOE may show more atrial thrombi than previously reported in cryptogenic and lacunar strokes was not confirmed by the results of this study. The frequency of thrombi was similar to those reported in studies which allowed a longer time between stroke and TOE, ${ }^{58}$ or was even lower. ${ }^{6}{ }^{28}$ Although our selection criteria were different from those employed in other studies, especially regarding the age limits and the exclusion of recurrent strokes, the size of our group seems large enough to allow valid results.

The findings about spontaneous echo contrast are of uncertain relevance. The similar frequency of SEC in cryptogenic and in lacunar strokes does not suggest a strong link with the aetiology of the recent ischaemia. All our patients with SEC received antiplatelet agents for secondary prophylaxis, and none experienced any ischaemic recurrence over a mean follow up of 25.8 months (range 18-36 months). Thus no definite negative prognostic value can be assigned to SEC in this very small subgroup. Other authors have found no evidence of SEC in cryptogenic strokes, ${ }^{6-8}$ a very low frequency, ${ }^{29}$ or a frequency higher than ours. ${ }^{5}$ Different selection criteria or small sample size may explain discordant results. Although SEC has been linked to the presence of atrial thrombi, ${ }^{15}$ to a history of arterial embolism, ${ }^{16}{ }^{17}$ and to stroke recurrence in one prospective series, ${ }^{30}$ some authors have found no association between the presence of SEC and the occurrence of ischaemic events in the follow up. ${ }^{31}$ In general, SEC seems associated with atrial thrombi and ischaemic events in patients with large atria, rheumatic mitral valve disease, valve prostheses, or atrial fibrillation. ${ }^{15-17}{ }^{30}$ The last three conditions were specifically excluded from our series.

In this study we excluded patients with one of the accepted causes of cardioembolism, ${ }^{27}$ because they will be given oral anticoagulants irrespective of whether they have an atrial thrombus or not. Conversely, very old, severely disabled, or demented patients were excluded because they are usually not treated with anticoagulants, due to the bleeding risk connected with advanced age and poor control of treatment. We also excluded patients with recurrent strokes because they probably harbour cardioembolic conditions more often than patients with a first stroke. So called "young strokes" were excluded as well, because the aetiology of brain ischaemia is more varied in this group, ${ }^{32}$ possibly with a greater contribution from the heart. Thus the results of heart investigations in young patients may not be directly applicable to older subjects. Using these criteria we tried to investigate patients with a similar chance of having cardiac sources of embolism, who could have actually been anticoagulated if a thrombus had been found.

Perhaps the inclusion of vertebrobasilar strokes decreased the percentage of positive findings, as it is always difficult to determine the aetiology of a posterior circulation stroke. Therefore, it is possible that many of our brainstem and cerebellar infarctions were due to intracranial atherosclerotic occlusion that was not detected by ultrasound. However, even excluding these patients, the frequency of atrial thrombi remains low.

Although a large fraction of eligible patients was not studied, we think that our results accurately reproduce the pattern of an unselected stroke population, as all missed cases were due to reasons unrelated to early stroke recurrence in hospital or to the potential mechanisms of 
stroke (such as vascular territory, coexisting diseases, cerebrovascular risk factors).

Despite the negative results, we think that this study may bring some useful information to clinicians involved in the early management of patients with a first stroke or transient ischaemic attack. As very early TOE does not seem to increase thrombus detection, this investigation may be delayed for some days, until the neurological deficit has stabilised, or patients are less hampered by their deficits, and may better tolerate the examination. This delay may also avoid unnecessary overloading of the cardiovascular laboratory schedule. Furthermore, any treatment may be initiated without the doubt that an early TOE may have substantially changed therapeutic decisions. Thus our results concur with the guidelines for early stroke management recently formulated in the United States ${ }^{33}$ and Europe ${ }^{34}$ in showing that it is not necessary to include TOE among the investigations during the very first days after a cryptogenic or lacunar stroke.

Our findings, and those of others, seem to show that the main yield of TOE in first crytpogenic strokes is represented by patent foramen ovale, ${ }^{9}$ atrial septal aneurysms, ${ }^{12}$ aortic plaques, ${ }^{14}$ and perhaps valve strands. ${ }^{35}$ Whether it is justified to look for these abnormalities in all such patients, in the absence of specific treatments, is unclear. ${ }^{29}$ This issue may be settled by prospective studies investigating the prognostic relevance of these findings in large series of patients.

1 Sacco RL, Ellenberg JH, Mohr JP, et al. Infarcts of undetermined cause: the NINCDS stroke data bank. Ann Neuro 1989;25:382-90.

2 Bogousslavsky J, Van Melle G, Regli F. The Lausanne stroke registry. Analysis of 1000 consecutive patients with first stroke. Stroke 1988;19:1083-92.

3 Lindgren A, Roijer A, Norrving B, et al. Carotid artery and heart disease in subtypes of cerebral infarction. Stroke 1994;25:2356-62.

4 Pop G, Sutherland GR, Koudstaal PJ, et al. Transesophageal Pop G, Sutherland GR, Koudstaal PJ, et al. Transesophageal sources in patients with transient ischemic attacks. Stroke sources in patient

5 Albers GW, Comess KA, DeRook FA, et al. Transesophageal echocardiographic findings in stroke subtypes. Stroke 1994;25:23-8.

6 Rauh G, Fischereder M, Spengel FA. Transesophageal echocardiography in patients with focal cerebral ischemia of unknown cause. Stroke 1996;27:691-4.

7 Cujec B, Polasek P, Voll C, et al. Transesophageal echocardiography in the detection of potential cardiac sources of embolism in stroke patients. Stroke 1991;22. 727-33.

8 Lee RJ, Bartzokis T, Yeoh T-K, et al. Enhanced detection of intracardiac sources of cerebral emboli by transesophageal echocardiography. Stroke 1991;22:734-9.

9 Ranoux D, Cohen A, Cabanes L, et al. Patent foramen ovale: is stroke due to paradoxical embolism ? Stroke 1993;24:314.

10 Homma S, Di Tullio MR, Sacco RL, et al. Characteristics of patent foramen ovale associated with cryptogenic stroke.
11 Cabanes L, Mas JL, Cohen A, et al. Atrial septal aneurysm and patent foramen ovale as risk factors for cryptogenic strokes in patients less than 55 years of age. Stroke 1993;24: $1865-73$.

12 The French Study Group on Patent Foramen Ovale and Atrial Septal Aneurysm. Recurrent cerebrovascular events in patients with patent foramen ovale, atrial septal aneurysm, or both and cryptogenic stroke or transient ischemic attack. Am Heart f 1995;130:1083-8.

13 Amarenco P, Cohen A, Tzourio C, et al. Atherosclerotic disease of the aortic arch and the risk of ischemic stroke. $N$ Engl f Med 1994;331:1474-9.

14 Tunick PA, Rosenzweig BP, Katz ES, et al. High risk for vascular events in patients with protruding aortic atheromas: a prospective study. F Am Coll Cardiol 1994;23:1085-90.

15 Black IW, Hopkins AP, Lee LCL, et al. Left atrial spontaneous echo contrast: a clinical and echocardiographic analysis. I Am Coll Cardiol 1991;18:398-404.

16 Daniel WG, Nellessen U, Schroeder E, et al. Left atrial spontaneous echo contrast in mitral valve disease: an indicator for an increased thromboembolic risk. $7 \mathrm{Am}$ Coll Cardiol 1988;11:1204-11.

17 Chimowitz MI, DeGeorgia MA, Poole RM, et al. Left atrial spontaneous echo contrast is highly associated with spontaneous echo contrast is highly associated with
previous stroke in patients with atrial fibrillation and mitral previous stroke in patients with a

18 Schneider B, Hofmann T, Justen MH, et al. Chiari's network-normal anatomic variant or risk factor for arterial embolic events ? f Am Coll Cardiol 1995;26:203-10.

19 Daniel WG, Mugge A. Transesophageal echocardiography. N Engl f Med 1995;332:1268-79.

20 Freedberg RS, Goodkin GM, Perez JL, et al. Valve strands are strongly associated with systemic embolization: a transesophageal echocardiographic study. $7 \mathrm{Am}$ Coll Cardiol 1995;26:1709-12.

21 Jones EF, Kalman JK, Calafiore P, et al. Proximal aortic atheroma. An independent risk factor for cerebral ischemia. Stroke 1995;26:218-24.

22 Hofmann T, Kasper W, Meinertz T, et al. Echocardiographic evaluation of patients with clinically suspected arterial emboli. Lancet 1990;336:1421-4.

23 Fieschi C, Argentino C, Lenzi GL, et al. Clinical and instrumental evaluation of patients with ischemic stroke within the first six hours. F Neurol Sci 1989;91:311-22.

24 Bogousslavsky J, Van Melle G, Regli F, et al. Pathogenesis of anterior circulation stroke in patients with nonvalvular anterior circulation stroke in patients with
atrial fibrillation. Neurology 1990;40:1046-50.

25 Whisnant JP, Basford JR, Bernstein EF, et al. Classification of cerebrovascular diseases III. Stroke 1990;21:637-76.

26 Coté R, Hachiski VC, Shurvell BL, et al. The Canadian neurological scale: a preliminary study in acute stroke. Stroke 1986;17:731-7.

27 Adams HP, Bendixen BH, Kappelle LJ, and the TOAST Investigators. Classification of subtype of acute ischemic stroke. Stroke 1993;24:35-41.

28 Stollberger C, Brainin M, Abzieher F, et al. Embolic stroke and transesophageal echocardiography: can clinical parameters predict the diagnostic yield ? F Neurol 1995;242:43742

29 Warner MF, Momah KI. Routine transesophageal echocardiography for cerebral ischemia. Is it really necessary ? Arch Intern Med 1996;156:1719-23.

30 Leung DJ, Black IW, Cranney GB, et al. Prognostic implications of left atrial spontaneous echo contrast in nonvalvular atrial fibrillation. $\mathcal{f} \mathrm{Am}$ Coll Cardiol 1994;24:755-62.

31 Archer SL, James KE, Kvernen LR, et al. Role of transesophageal echocardiography in the detection of left atrial thrombus in patients with chronic nonrheumatic atrial fibrillation. Am Heart 7 1995;130:287-95.

32 Adams HP, Kappelle LJ, Biller J, et al. Ischemic stroke in young adults: experience in 329 patients enrolled in the lowa registry of stroke in young adults. Arch Neurol 1995;52:491-5.

33 Adams HP, Brott TG, Crowell RM, et al. Guidelines for the management of patients with acute ischemic stroke. Stroke 1994;25:1901-14

34 The European Ad Hoc Consensus Group. European strategies for early intervention in stroke. Cerebrovasc Dis 1996;6:315-24.

35 Cohen A, Tzourio C, Chauvel C, et al. Mitral valve strands and the risk of ischemic stroke in elderly patients. Stroke 1997;28:1574-8. 